\title{
Cloud intelligence in manufacturing
}

\author{
Tin-Chih Toly Chen ${ }^{1}$
}

Received: 19 June 2015 / Accepted: 30 June 2015 / Published online: 8 July 2015

(C) Springer Science+Business Media New York 2015

Cloud manufacturing (CMfg) is a new-generation, centrally-managed, service-oriented networked manufacturing model that can provide manufacturing resources and services to users in different locations (Wu et al. 2013b; Archimede et al. 2014). Cloud computing enthusiasts have advocated CMfg as a direct extension of cloud computing in the manufacturing sector, but the full application of cloud computing to manufacturing is a problem (Chen 2014). There are also many differences between information services and manufacturing services (Fan et al. 2004). Nevertheless, from the existing reports and case studies, the benefits of CMfg appear to include: cost savings, efficiency, additional data analysis capabilities, flexibility, and closer partner relationships (Chen 2014). While CMfg may be suitable for small or mid-sized enterprises, most capital-intensive businesses directly buy all necessary systems and equipment (Chen 2014), which makes some of the cost-saving incentives insignificant. Many manufacturing operations managers have hesitated to migrate systems to clouds (Davidson 2013). Although some researchers have asserted that CMfg is a new paradigm that will revolutionize manufacturing $(\mathrm{Wu}$ et al. 2014), most existing CMfg technologies center on information technology, rather than manufacturing technology (Chen 2014). To tackle these problems, manufacturers need manufacturing-oriented CMfg, supplemented by information technology.

In the opinion of $\mathrm{Wu}$ et al. (2013a), research fields critical to the enablement of CMfg include automation, industrial control systems, service composition, flexibility,

\section{Tin-Chih Toly Chen}

tcchen@fcu.edu.tw

1 Department of Industrial Engineering and Systems Management, Feng Chia University, 100, Wenhwa Road, Seatwen, Taichung City 407, Taiwan business models, implementation models, and architectures. In addition, two important concepts in cloud computing interoperability and scalability - if successfully applied to CMfg, can enable flexible responses to orders and flexible adjustments of factory capacity (Newman et al. 2008; Panetto and Molina 2008; Chen 2014; Galasso et al. 2014). The mainstream research on CMfg has been focused on topics like cyber-physical systems (CPSs) (Colombo et al. 2013), resource virtualization and visualization (Chen and Romanowski 2014; Huang et al. 2015), cloud-based manufacturing services (Wang and Xu 2013), distant monitoring and control, and others. Entirely new directions for CMfg, including Industry 4.0 (Federal Ministry of Education and Research 2013), are yet to be developed.

The objective of this special issue is intended to provide the details of developing manufacturing-oriented CMfg intelligence and its applications for researchers in process engineering, industrial engineering, information engineering, and operations research, as well as practicing managers/engineers. This special issue features a balance between state-of-the-art research and usually reported applications. This special issue also provides a forum for researchers and practitioners to review and disseminate quality research work on manufacturing-oriented CMfg intelligence and its applications, and to identify critical issues for further developments. After a strict review, eleven articles from researchers around the world were finally accepted.

Since 1960s, with the development of societal and related technologies, many advanced manufacturing systems (AMSs) and modes have been put forward. F. Tao, Y. Cheng, L. Zhang, and A. Y. C. Nee overviewed the development process of AMSs, and established a tri-view model to analyze the evolution and socialization characteristics of AMSs. It is found that the sharing of manufacturing resources and capabilities, the value creation carriers, the value measuring 
criteria, the composition of the value chain and enterprise collaboration, and user participation in manufacturing are all moving towards socialization.

Factory operations have shifted from labor-based to semi-automatic and fully automatic and may even become unmanned in the future. Therefore, the conditions of a factory can be monitored from a distance and the machines can be remotely controlled. T. Chen, Y.-C. Wang, and Z. Lin investigated the predictive distant operation of a computer numerical control (CNC) machine virtually controlled with hand gestures. The attempt in their study is a crucial step toward entirely Internet- or cloud-based manufacturing.

Y. Zhang, G. Zhang, Y. Liu, and D. Hu presented a service encapsulation and virtualization access model for manufacturing machines by combining the IoT (Internet of Things) techniques and cloud computing. By implementing the proposed service encapsulation and virtualization access model, the capability of a machine could be actively perceived, the production process is transparent and can be timely visited, and the virtualized machine could be accessed through a loose coupling, 'plug and play' manner.

I. Nielsen, Q.-V. Dang, G. Bocewicz, and Z. Banaszak implemented an autonomous industrial mobile robot in realworld industrial applications. The proposed methodology consists of a mobile robot system design (Little Helper prototype), an appropriate industrial application (multiple-part feeding), an implementation concept for the industrial application (the Bartender concept), a mathematical model, and a genetic algorithm (GA)-based heuristic. In addition, in order for a mobile robot to work properly in a cloud-based manufacturing environment, the communication and exchange of data between the mobile robot with other manufacturing systems and shop-floor operators were addressed.

Computing resource allocation in a cloud manufacturing system is a NP-hard problem. Y.-K. Lin and C. S. Chong presented a GA-based resource constraint project scheduling method for solving this problem. In the proposed methodology, conflict resolutions and enhancements were performed on newly generated offsprings after crossover or mutation. Then, the local search exploited the neighborhood of solutions to find better schedules.

J. Wang, L. Zhang, L. Duan, and R. X. Gao investigated a cloud-based paradigm of predictive maintenance based on mobile agents. In the paradigm, a low-cost cloud sensing and computing node was developed with the embedded Linux operating system, a mobile agent middleware, and open source numerical libraries. Information sharing and interaction was achieved by mobile agents through distributing the analysis algorithm to the cloud sensing and computing node.

Estimating the cycle time of each job in a wafer fabrication factory is critical. Recently, a few researchers have attempted to establish the asymmetric bounds of a cycle time. However, these methods either have overly complex computations or do not consistently perform well. T. Chen and H. C. Wu proposed a new approach for effective cycle-time bounding. They first constructed a back propagation network to predict the cycle time of a job. Then, two linear functions of the output were formed, and the threshold on the output node was fuzzified to derive the lower and upper bounds of the cycle time. In addition, a cloud computing scheme is proposed to improve the bounds in an effective manner.

An essential and practical application of cloud manufacturing is factory simulation as a cloud service (FSaCS). T. Chen and C. W. Lin discussed several topics related to implementing FSaaCS. Among them, load balancing was considered a critical topic. To address this topic, they summarized factors critical to estimating a simulation workload, and applied several methods to estimate a simulation load, relevant to the required simulation time, from these factors.

Cloud computing enables many applications of Web services and rekindles the interest of providing design services via the Internet. Recent research indicates that design delivered thru could computing will outperform the traditional IT offers. C.-C. Huang, W.-Y. Liang, and S.-R. Yi provided a total solution that was able to model the relationship of components and modularity, explore component disassemblability and identify modules, recognize disassembly patterns, provide disassembly guidelines and recyclable component classification to instruct how to disassemble components. In addition, based on a cloud computing architecture, designers exchanged and stored their design information and knowledge for new sustainable product developments.

Most relevant studies on virtualization have attempted to separate software packages, computing, and data storage from physical hardware. Other manufacturing resources (e.g., equipment) that cannot be easily virtualized and placed in a public pool were investigated by T. Chen and Y. C. Lin. They proposed an innovative information resource and service, a digital equipment identifier (DEI) system. The DEI system is a new identification scheme that assigns each piece of equipment a unique identification, based on which various applications can be practiced.

Cloud-based design for configurations (CBDC) can be referred to as a service-oriented networked design for configuration models. However, cloud-based models also pose challenges such as reliability, availability, capability, ability, adaptability of resources, and services across spatial boundaries. Multi-scale design can presumably stimulate greater intelligence in cloud-based models. Using the concepts of the fuzzy holon and the fuzzy attractor, H. Issa, E. Ostrosi, M. Lenczner, and R. Habib proposed the fuzzy holonic approach to address multi-scale design for configurations.

I would like to thank to the JIMS Editor-in-Chief Andrew Kusiak for providing full support in bringing out this special issue. I am thankful to the paper contributors who shared their research as well as the reviewers who spared their 
valuable time in paper review. I would also like to thank the Journal staff. Without their support and professional assistance, the prepublication process would not have been possible.

\section{References}

Archimede, B., Letouzey, A., Memon, M. A., \& Xu, J. (2014). Towards a distributed multi-agent framework for shared resources scheduling. Journal of Intelligent Manufacturing, 25(5), 1077-1087.

Chen, T. (2014). Strengthening the competitiveness and sustainability of a semiconductor manufacturer with cloud manufacturing. Sustainability, 6, 251-268.

Chen, T., \& Romanowski, R. (2014). Forecasting the productivity of a virtual enterprise by agent-based fuzzy collaborative intelligence-With Facebook as an example. Applied Soft Computing, 24, 511-521.

Colombo, A. W., Bangemann, T., Karnouskos, S., Delsing, J., Stluka, P., Harrison, R., et al. (2013). Industrial cloud-based cyber-physical systems-The IMC-AESOP approach. Switzerland: Springer International Publishing.

Davidson, M. (2013). Why cloud manufacturing software adoption rates are rising. https://www.manufacturing.net/articles/2013/10/ why-cloud-manufacturing-software-adoption-rates-are-rising

Fan, Y., Zhao, D., Zhang, L., Huang, S., \& Liu, B. (2004). Manufacturing grid: Needs, concept and architecture. Lecture Notes in Computer Science, 3032, 653-656.
Federal Ministry of Education and Research. (2013). Project of the future: Industry 4.0. http://www.bmbf.de/en/19955.php

Galasso, F., Ducq, Y., Lauras, M., Gourc, D., \& Camara, M. (2014). A method to select a successful interoperability solution through a simulation approach. Journal of Intelligent Manufacturing. doi:10.1007/s10845-014-0889-4

Huang, H.-C., Lin, Y.-C., Hung, M.-H., Tu, C.-C., \& Cheng, F.-T. (2015). Development of cloud-based automatic virtual metrology system for semiconductor industry. Robotics and ComputerIntegrated Manufacturing, 34, 30-43.

Newman, S. T., Nassehi, A., Xu, X. W., Rosso, R. S. U., Wang, L., Yusof, Y., et al. (2008). Strategic advantages of interoperability for global manufacturing using $\mathrm{CNC}$ technology. Robotics and Computer-Integrated Manufacturing, 24(6), 699-708.

Panetto, H., \& Molina, A. (2008). Enterprise integration and interoperability in manufacturing systems: Trends and issues. Computers in Industry, 59(7), 641-646.

Wang, X. V., \& Xu, X. W. (2013). An interoperable solution for cloud manufacturing. Robotics and Computer-Integrated Manufacturing, 29(4), 232-247.

Wu, D., Greer, M. J., Rosen, D. W., \& Schaefer, D. (2013). Cloud manufacturing: Strategic vision and state-of-the-art. Journal of Manufacturing Systems, 32(4), 564-579.

Wu, D., Thames, J. L., Rosen, D. W., \& Shcaefer, D. (2013). Enhancing the product realization process with cloud-based design and manufacturing systems. Journal of Computing and Information Science in Engineering, 13, 041004-1-041004-14.

Wu, D., Rosen, D. W., Wang, L., \& Schaefer, D. (2014). Cloud-based manufacturing: Old wine in new bottles? Procedia CIRP, 17, 9499. 